\title{
Reducing non-attendance rates in a community mental health team
}

\author{
Maria Filippidou, Susie Lingwood, llyas Mirza
}

Barnet, Enfield \& Haringey Mental Health Trust

\begin{abstract}
The project aimed to improve productivity of psychiatric out patient clinic using quality improvement techniques through "Listening Into Action", a national programme designed to engage and support front-line clinicians to make improvements to patient care. We identified reasons as to why our patients missed appointments and then introduced a system to reduce "did not attend" (DNA) rates.

Non-attendance at appointments results in a waste of resources and increases waiting times. It has been reported that DNA rates in mental health are higher compared to other settings. Therefore, reducing DNA rates are a priority for mental health care providers.

We collected DNA rates over a period of months over May 2013 to September 2013. We conducted a patient survey to inquire why the patients missed their appointments. The aim of the project and results from the survey were presented and discussed at the multi-disciplinary team meeting to generate ideas for improvement and engage the team with the project. As the most frequent response from the survey was 'forgetting the appointment', we decided to introduce text messaging as an intervention to remind patients of their appointments. We also ensured that staff updated the mobile phone records for the patients at each appointment. We monitored the DNA rates after introducing this change on a monthly basis.
\end{abstract}

Following our intervention, there was an overall reduction in DNA rates for all disciplines from $11.4 \%$ to $10.62 \%$ with the greatest change for medical DNA's from $17.7 \%$ to $11.8 \%$.

Results from a patient survey showed that the reasons for non-attendance are multi-factorial and require a complex approach. Our intervention was a simple one but still it demonstrated some effectiveness. Reducing DNA rates requires interventions to be regularly monitored so that their effect is sustained over a period of time.

\section{Problem}

This project was undertaken at a community mental health team in North London, UK. It was one of the first 10 projects of Listening into Action in the Trust, a national programme designed to engage and support front-line clinicians to make improvements to patient care.

According to Enfield Council data[1], Enfield is the 64th most deprived local authority district in the UK. The total population of the borough was 312,466 according to the 2011 census. According to the same census, there was a $60 \%$ increase in the non-UK born population, mainly from Turkey, Poland, Nigeria, Ghana, and Somalia. There was also an increase of $71 \%$ in lone parents with children dependent to them. While unemployment in London went up by $38 \%$ since the last census in 2001, in Enfield it rose by $61 \%$.

Non-attendance of outpatient/community appointments has been a long-standing issue for the NHS and this was also the case in our team. New and follow up appointments were being missed. Many community teams manage non-attendance by discharging patients who miss consecutive appointments. As a consequence, these patients may fail to see a specialist when needed and thereby impacting on the continuity of care, compliance with medication, and engagement with the service. This translates into a financial impact on the service and the professionals' morale.

\section{Background}

It is estimated that around $£ 360$ million per year is lost due to nonattendance of appointments in the NHS.[2] According to Mitchell \& Selmes [3], this rate of non-attendance for psychiatric appointments is double the one for other medical specialties. According to the Department of Health, in 2002-2003 the did not attend (DNA) rate of psychiatric outpatient appointments was $19.1 \%$ in England, while the overall NHS rate was $11.7 \%$.[3-4]

Clinical evidence suggests that psychiatric patients who miss follow up appointments have a higher chance of being admitted over a 12-month period and there is more likelihood of a negative effect on the condition.[5]

Non-attendance in outpatient clinics across all specialties has been extensively studied. Akhter et al (2012) used telephone survey methodology to elicit for reasons of non-attendance in a diabetes clinic. [6] Their main finding was that forgetting the appointment was the most frequent response and suggested that simple administrative steps have a role. Guy et al (2012) in a systematic review and meta analysis on the effectiveness of text messaging as a reminder of appointments in the health care setting [7], found that text messaging increases attendance and the method was thought to be a simple and efficient one. Similarly, Sims et al (2012) studied the effect text messaging at four community mental health clinics in 
London.[8] They found that a reduction of $25-28 \%$ happened post implementation and concluded that such an effect could save roughly £150 million on a national level in England. Paterson et al (2010) in their literature review on the subject of non-attendance in chronic disease clinics showed that the issue was multifactorial and that there are system- and person- related factors that need to be addressed if a solution is to be found.[9]

Other methods that have been studied and have been found to be successful in reducing non-attendance are email reminders [10], having an opt-in system in place where the patient has to respond is some way to the invitation for an appointment [11], and "patient focused booking" no more than six weeks in advance.[12]

\section{Baseline measurement}

Baseline DNA rates were collected from the community mental health team. The team consisted of three basic disciplines: doctors, psychologists, and care coordinators (nurses and social workers) but underwent a few changes during the months of study. The project looked at changes in DNA rates for the new and follow up appointments in all three disciplines between the months of May 2013 to March 2014. The junior doctor's (CT) appointment data were excluded from the study because their presence was not consistent throughout the year.

Data were obtained automatically from the electronic database system (RiO). The difference between "cancelled" and "did not attend" was that for the former patients would call us to give a reason for not being able to attend, whereas they wouldn't make any contact at all prior to the appointment for the latter. For the purpose of this quality improvement project, we only studied the "did not attend" rates.

We devised an anonymous questionnaire with closed questions along with free text questions. These focused on the reasons for missing appointments and what could be done to help them not miss appointments in the future.

In terms of DNA rates, baseline measurements were taken for the months of May, June, July, August, and September 2013. These were later grouped (group A) as the set of measurements prior to implementation of our intervention. Individual and overall rates can be seen on tables 1 and 2 .

See supplementary file: ds3157.docx - "Survey questionnaire"

\section{Design}

Following the analysis of the data, the patient survey results, and the team discussion on the issue, it was evident that team DNA rates were similar to other teams in the trust. If our quality improvement project demonstrated reduction in rates, then this could be adapted an implemented in other parts of the trust to improve efficiency. We found out that the most common reason for not attending an appointment from the perspective of the patient was forgetting about it. Patients' main suggestion through the survey was to have a system of reminding them via texts or phone calls prior to the appointment. Another suggestion from the team had to do with problems identified on our electronic system. They referred to the incomplete drop down menu when they had to record a non-attendance and came up with ideas like adding the options of "contacted carer" or "made unannounced visit" when a patient would miss their appointment.

Suggestions for our electronic system were passed on to the relevant team (RiO steering group). They noted our suggested and stated that it would be implemented when our system will be managed by another provider in 2015-2016. The current system did not allow the trust flexibility to make changes.

With regards to the initial suggestion of reminding our patients of their appointment, we took some steps towards activating text messaging in our team as the service already existed in the trust. The text messages would be delivered three days and one day prior to the appointment. Another step taken was to ask all members of staff to update our patients' mobile phone numbers on the electronic system each time they met.

\section{Strategy}

Following literature search to look at what has been studied in terms of DNA rates in mental health clinics, PDSA cycle 1 was performed. Although there was some very good information on the possible reasons why patients miss their appointments, it wasn't clear whether results could be generalised to our population. PDSA cycle 1 took place where a questionnaire anonymously given to patients attending our clinic over a period of a month was designed. The questions were focused on possible reasons why individuals do not attend their appointments and there was also free text for them to write their ideas with regards to this, as well as possible ways their attendance could improve. A 2nd PDSA cycle took place where a meeting with the team was held for their opinions on the issue to be heard. Some very interesting ideas came out of this meeting; it raised staff awareness on helping patients to engage better but also record their appointment outcomes on the system more appropriately. Following that meeting, it was agreed that a realistic intervention would be to introduce the text messaging system. A 3rd PDSA was performed along this idea, which showed some very positive results.

See supplementary file: ds3962.docx - "PDSA Cycles (1)-2"

\section{Post-measurement}

October 2013 was the first month of results following our intervention. The overall DNA rate for the service had reduced to $7.5 \%$. The rates remained equally low in November 2013 but there was an increase in December 2013 (though still lower rate since implementation of intervention). In January 2014, the rates started decreasing again but not as dramatically as before and in February 2013 the rate was $11.9 \%$.

DNA rates were also studied after the implementation for the three 
separate disciplines of the team. These were also grouped (Group B) and overall rates were calculated. We considered statistical tests to test significance of the results but we decided not to use them for several reasons that are discussed later.

Looking at different disciplines, the most prominent fluctuation happened within the psychology team. An explanation for this would be the fact that psychological interventions require patients to attend appointments on a weekly basis at our premises. This can appear quite inflexible for the community mental health patients with a variable degree of engagement. This expectation to attend regularly serves a significant therapeutic purpose with regard to boundary setting and containment, but may be the reason for the observed fluctuations.

With regards to care coordination, an increase in DNA rates took place during the months of December 2013 and January 2014. It is a common experience for mental health services but also other healthcare clinics to have an increase in DNA rates during these festive months. Reasons for this have to do with both patients but also absences of members of staff. Another significant reason for fluctuations within the group of care coordinators has been the extensive changes in members of staff within our team. Finally, the medical absolute DNA rates decreased consistently since intervention, in accordance with the overall consistent reduction. However, there was also a relative increase that took place during the months of December 2013 and January 2014.

Results can be viewed in the form of a run chart on figures 1 and 2 . The number of contacts with patients in the team can be seen on figures 3 and 4 , which gives a perspective of the workload of the team.

See supplementary file: ds3835.pdf - "Results"

\section{Lessons and limitations}

What was appreciated in this project was how important it is to have a very focused question/ problem. That increases the chances of the project being successful in the sense of bringing about a change. It was also important to engage with all stakeholders to obtain different perspectives possible resolutions.

The lack of statistical testing is a limitation. However, this was a service improvement project, not a research project that needed power calculations and a sample size with enough power to detect a difference. We did not want to give the erroneous impression of significance where there was none, and decided that it would be best that the effect of the intervention be described in rates so that variance can be seen and an impression of the effect size can be made.

With regards to this project, there is certainly room for improvement. It is important to continue working on it for the months or years to come, implementing different methods of engaging patients with treatment. We would also suggest extending the survey that was done with regards to patient's views on missing appointments, reasons and methods for improvement. Once we have robust conclusions, the same methods can be used in other teams of the trust. Finally, results need to be disseminated to the team and other stakeholders regularly.

\section{Conclusion}

The problem identified prior to this project was the DNA rates and the differences between the disciplines with regards to this. A team meeting was organised where opinions were sought from members of staff and key the stakeholders identified. A self reported questionnaire was designed and given to the patients. The questionnaire sought their opinions on why they miss appointments and what they think would improve their engagement. Finally, we recorded the baseline team DNA rates through the electronic system.

Following that process, it was decided that the intervention should be the introduction of text messaging prior to appointments. Other suggested changes were altering the recording methods of appointment outcomes on the electronic system. There was a dramatic fall of DNA rate in the month following the implementation of the intervention. The fall in DNA rates leveled off during the months that followed, but it remained lower than the baseline rates, ie pre-intervention rate. Different reasons for this shift and factors that could have temporarily increased the rates were explore such as changes in the members of staff within the team.

Finally, we took steps with regards to the sustainability of the project. This was done by consistently educating staff on the significance of reminding patients of their appointments. The project was handed over to other members of the team to continue with monitoring monthly DNA rates and discussion of how these can be improved further.

\section{References}

1. Enfield Council, 2014, Census 2011, Retrieved from www.enfield.gov.uk.

2. Stone CA, Palmer JH, Saxby PJ, Devaraj VS. Reducing nonattendance at outpatient clinics. J R Soc Med 1999. 92(3):114.

3. Mitchell A. J. and Selmes, T., Why don't patients attend their appointments? Maintaining engagement with psychiatric services, Advances in Psychiatric Treatment, 2007, 13: 423-34.

4. Department of Health (2003) Hospital Activity Statistics, London. Department of Health.

5. Killaspy H, Banerjee S, King M, Lloyd M. Prospective controlled study of psychiatric out-patient non-attendance. Characteristics and outcome. Brit J Psych 2000. 176:160-5.

6. Akhter K, Dockray S, Simmons D. Exploring factors influencing non-attendance at the diabetes clinic and service improvement strategies from patients' perspectives. Practical Diabetes 2012. 29(3):113-6.

7. Guy R, Hocking J, Wand H, Stott S, Ali A, Kaldor J. How Effective Are Short Message Service Reminders at Increasing Clinic Attendance? A Meta-Analysis and 
Systematic Review. Health Serv Res 2012, 47(2):614-32.

8. Sims H, Sanghara H, Hayes D, Wandiembe S, Finch M, Jakpbsen H, Tsakanikos E, Okocha Cl, Kravariti E. Text Message Reminders of Appointments: A Pilot Intervention at Four Community Mental Health Clinics in London. Psych Serv 2012. 63(2):161-8.

9. Paterson B, Charlton P, Richard S. Non-attendance in chronic disease clinics: a matter of non-compliance? J Nursing Health Chron III, 2010, 2:63-74.

10. Lim L, Varkey P. E-mail Reminders: A Novel Method to Reduce Outpatient Clinic Nonattendance. Internet J Health Admin 2004. 3:1.

11. Hawker DSJ. Increasing initial attendance at mental health out-patient clinics: opt-in systems and other interventions. Psychiatr Bull 2007. 31:179-82.

12. Milne RG. Reducing non-attendance at specialist clinics: an evaluation of the effectiveness and cost of patient-focussed booking and SMS reminders at a Scottish health board. Int J Consumer Stud, 2010, 34(5):570-80.

\section{Declaration of interests}

No competing interests.

\section{Acknowledgements}

We would like to thank Barbara Luker and Gary Oland for their valuable contribution. 